\title{
Temporary Prosthetic Shunt to Permanent Aortic Prosthesis in a Patient with an Infected Thoracoabdominal Aneurysm to Shorten Ischemia Time
}

\author{
Einar Dregelid", and Axel Algaard ${ }^{2}$ \\ ${ }^{1}$ Department of Vascular Surgery, Haukeland University Hospital, Jonas Lies vei 65, 5021 Bergen, Norway; Institute for \\ Surgical Sciences, University of Bergen, Jonas Lies vei 65, 5021 Bergen, Norway \\ ${ }^{2}$ Department of Orthopaedic Surgery, Haukeland University Hospital, Jonas Lies vei 65, 5021 Bergen, Norway
}

\begin{abstract}
High operative mortality of infected thoracoabdominal aortic aneurysms (ITAA) is partly attributable to ischemic injury during aortic clamping. We report a 62-year-old man with biliary cirrhosis, who developed a rapidly enlarging ITAA secondary to thoracolumbar osteomyelitis. Additional infectious foci were found in the pubic and ischial bones and in the left lung. Blood cultures gave growth of streptococcus pneumoniae. The aneurysm was repaired through a thoracoabdominal incision with a Dacron prosthesis. Prior to aneurysm repair, a prosthetic shunt was anastomosed end to- side to the aortic prosthesis and to the descending aorta using a side-biting clamp. The shunt allowed perfusion of the lower body and of renal and visceral vessels after 45 minutes, the time needed to resect infected tissue and complete the distal anastomosis. The proximal anastomosis and orthopedic treatment of the spinal osteomyelitis could be performed, while the lower body and visceral organs were perfused.

Postoperatively, the patient developed hypotension and increasing lactacidosis. Laparotomy revealed intestinal infarction, and gut resection was performed. Following a temporary improvement, he developed multiorgan failure and candida sepsis and died after 32 days. No atheroemboli were found in arteries of resected intestines. Portal hypertension most likely was present and it could be calculated that minimum intestinal perfusion pressure the night after the operation could have been in the range of 30-37 mm Hg, which probably was not enough to maintain aerobic metabolism. In the presence of aortic atheromas it may be advisable to divert blood to the shunt from an axillary artery.
\end{abstract}

Keywords: Thoracoabdominal aneurysm, infected aneurysm, ischemia, temporary bypass, arterial prosthesis, biliary cirrhosis.

\section{INTRODUCTION}

The operative mortality of infected thoracoabdominal aneurysms (ITAA) is in the $12-40 \%$ range [1-4], and a significant portion of the mortality is due to ischemic injury during aortic clamping [5]. Methods to avoid ischemic injury include the use of conventional extracorporal technology for perfusion of the lower body and selective perfusion of the visceral arteries $[6,7]$. With this method ipsilateral lower extremity ischemia and rhabdomyolysis-induced renal morbidity due to femoral artery cannulation has been a potential complication [7]. In patients with hemorrhagic diathesis or with infectious aneurysms with uncontrolled infection, although feasible, the use of conventional extracorporal circulation, as well as hypothermia, furthermore, may be less desirable due to the unfavorable effect on immune defense, and on hemostasis [8].

The objective of this paper is to show how aortic clamp time can be reduced by the time needed to suture the proximal anastomosis with the use of a temporary arterial prosthetic shunt to the permanent aortic prosthesis. The observations from a case with an infected thoracoabdominal aortic aneurysm are presented.

\footnotetext{
*Address correspondence to this author at the Department of Vascular Surgery, Haukeland University Hospital, Jonas Lies vei 65, 5021 Bergen, Norway; Tel: 047 91311797; Fax: 047 55972793;

E-mails: eidreg@yahoo.com, einar.dregelid@helse-bergen.no
}

\section{CASE PRESENTATION}

The case was a 62-year-old Caucasian man who was a long-time smoker of 10-15 cigarettes a day. He had been treated with NSAIDs, and intermittently with prednisolone for arthralgias and tendinitis for the last 15 years and with ursodeoxycholic acid for the last 4 years for primary biliary cirrhosis. While suffering from back pain for several months he lost weight from 88 to $73 \mathrm{~kg}$. At admittance he had also had pain in his right hip for a few weeks. CT examinations revealed an infectious thoracoabdominal aortic aneurysm, osteomyelitic destruction of the adjacent $12^{\text {th }}$ thoracic and $1^{\text {st }}$ lumbar vertebrae with sequestration (Figs. 1, 2), and osteomyelitis in the pubic, and ischial bones. There was an opacification in the left lung with pleural fluid. Blood cultures gave growth of streptococcus pneumoniae. Antibiotic treatment with Penicillin G and Clindamycin was begun and CRP fell, but then rose again and Clindamycin was substituted for Rifampin. The infectious aneurysm grew, causing excessive pain, and aneurysm surgery was planned. Risks and benefits of the proposed treatment were explained to the patient who gave informed consent.

Trabecular bone chips from the left iliac crest were harvested and stored for later transplantation. Sequestered bone was removed from the pubic bone. Three days later he underwent thoracolaparotomy. The left pleura was entered through the bed of the resected $10^{\text {th }}$ rib which was stored for transplantation. The lower thoracic and perirenal aorta was 


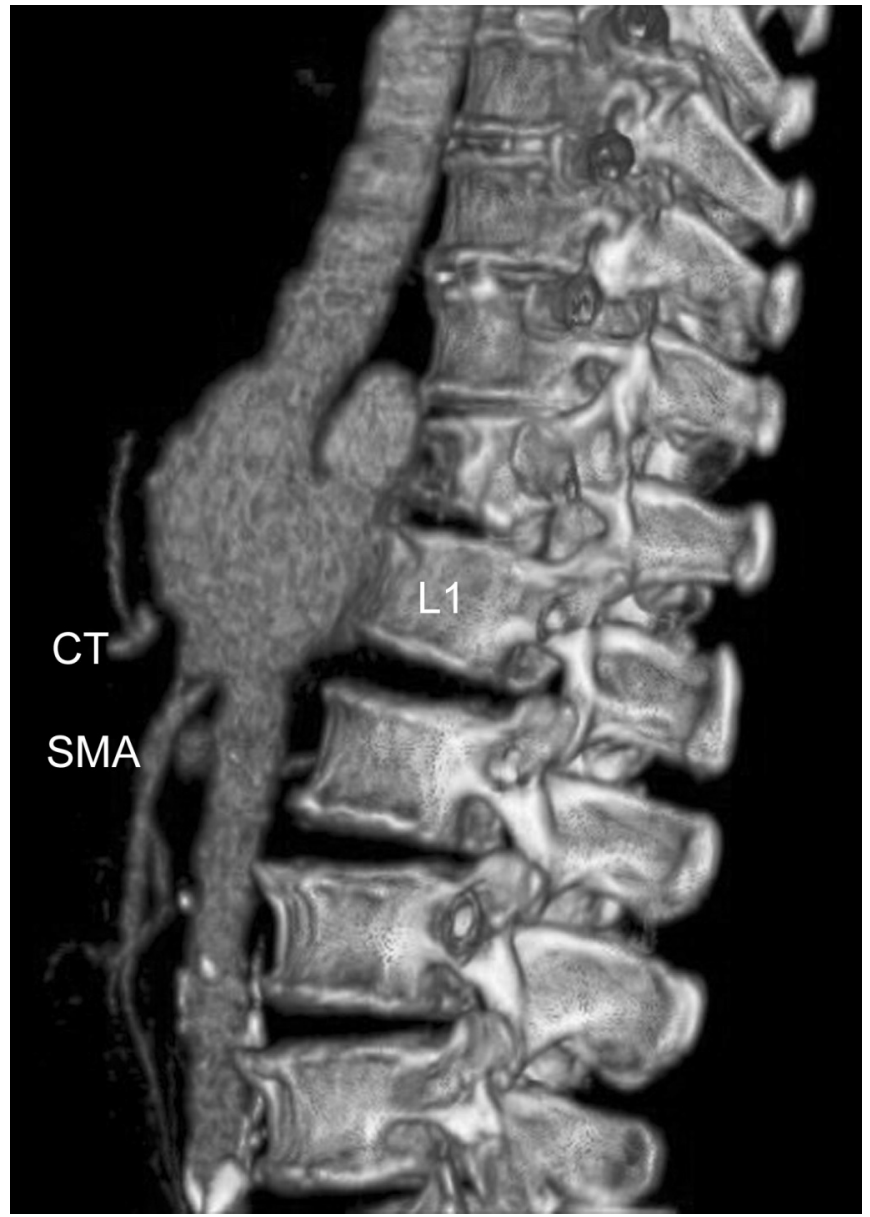

Fig. (1). Infectious thoracoabdominal aortic aneurysm affecting visceral arterial ostia. The aneurysm affected the first part of the celiac trunk (CT) and the upper margins of the superior mesenteric (SMA) and left renal arteries. There was osteolytic destruction of the adjacent $12^{\text {th }}$ thoracic and $1^{\text {st }}$ lumbar (L1) vertebrae with sequestration. This reformatted CT angiography shows only the blood-filled part of the aneurysm, not the mural thrombus.

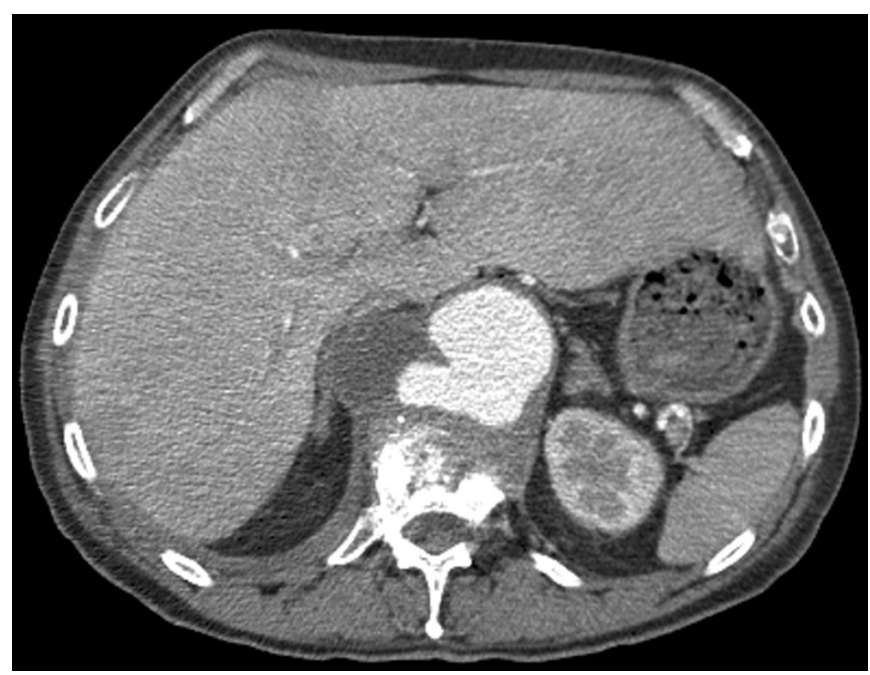

Fig. (2). Axial image of CT angiography shows the infectious thoracoabdominal aortic aneurysm.

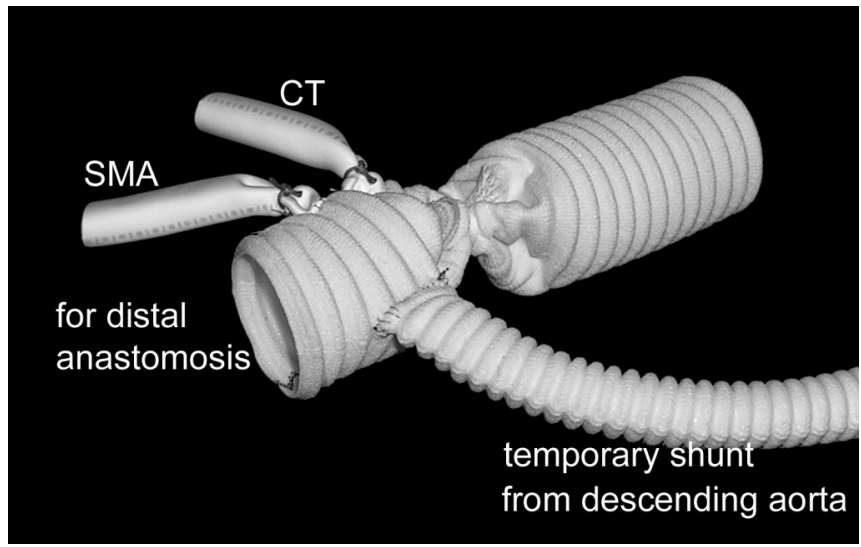

Fig. (3). Arterial prosthesis construct including temporary shunt and permanent aortic prosthesis used to treat the aneurysm. The vascular prosthesis construct included a $10 \mathrm{~mm}$ diameter temporary shunt from the descending aorta to the permanent aortic prosthesis. CT: side branch to celiac trunk. SMA: side branch to SMA; this was removed and the hole in the permanent prosthesis was oversewn since the SMA ostium could be included in the distal aortic anastomosis. To avoid cluttering the field by clamps, temporary ligatures were used to occlude both main graft and side branches. The side branches were ligated to the aortic graft (not shown) to prevent further cluttering during performance of the distal anastomosis. The three anastomoses and ligatures shown in the figure were tested for sufficient watertightness before the construct was used in the patient. A side-branch of the shunt (not shown) was forked into two coronary perfusion cannulas but these were not used due to cumbersomeness and space limitation. Also, the celiac ostium was stenotic, measuring only 1-2 $\mathrm{mm}$ in diameter and was not immediately identified.

exposed. A presutured vascular prosthesis construct (Fig. 3) that had been soaked in Rifampicin was anastomosed to the lower thoracic aorta using an exclusion clamp and then clamped close to the aorta. After heparinization (105 IU/kg body weight i.v.) and aortic clamping, the pseudoaneurysm was opened. The celiac ostium, the upper margin of the SMA ostium and the upper margin of the left renal artery were affected by the infection. Infected tissues were resected and the aortic graft of the vascular prosthesis construct was anastomosed to the distal aorta, including the remains of the left renal ostium and the anterior, partially resected, wall of the SMA in the anastomosis. Following completion of this anastomosis, the lower body, renal arteries, and SMA could be reperfused after 45 minutes of aortic clamping. Another part of the prosthesis construct, a 6-mm PTFE graft, was then anastomosed to the celiac artery after the infected and severely stenosed proximal part of it (1-2 mm lumen) had been resected. Infected bone and intervertebral disc were removed using a sharp spoon and a rongeur. Bone that had been removed three days earlier from the iliac crest and the chipped $10^{\text {th }}$ rib was transplanted to the resulting cavity. A gentamicin-impregnated collagen mattress, Gentacoll (Schering-Plough A/S, Farum, Denmark), was interposed between the bone transplant and the permanent aortic prosthesis which was then anastomosed to the thoracic aorta. The temporary bypass to the aortic prosthesis was removed, 
two drains were inserted into the left pleural cavity, and the wound was closed. The perioperative bleeding was $2500 \mathrm{ml}$.

Postoperatively the patient was initially stable and was extubated after 3 hours, but became unstable with a mean BP that was only $50 \mathrm{~mm} \mathrm{Hg}$ for altogether approximately 2 hours (Fig. 4). He had sinus tachycardia with a maximum frequency of 115 , and a respiratory frequency between 25 and 31 breaths/min. He became agitated, developed $\mathrm{CO}_{2}$ retention and was put on CPAP with PEEP $5 \mathrm{~cm} \mathrm{H}_{2} \mathrm{O}$. Urinary bladder pressure was $10 \mathrm{~mm} \mathrm{Hg}$. His blood pressure remained low despite volume substitution, dopamine (maximum dose: $40 \mu \mathrm{g} / \mathrm{min}$ ) and norepinephrine (maximum dose: $0.5 \mu \mathrm{g} / \mathrm{kg} / \mathrm{min}$ ). He was cold peripherally and urine production dropped. Serum lactate gradually rose to 7.4 $\mathrm{mmol} / \mathrm{L}$. His blood glucose was down to $2.1 \mathrm{mmol} / \mathrm{L}$. An abdominal CT angiography the day after the operation showed possible small intestinal infarction and a nonoccluding thrombus in the origin of the SMA. He therefore underwent laparotomy for removal of gangrenous tissues. The distal $1.5-\mathrm{m}$ of the small intestine was resected with end to end anastomosis, the sigmoid colon was resected and a colostomy performed, the gallbladder and part of the major omentum were removed. An infrarenal aorta-to-SMA PTFE bypass was constructed, but was probably not necessary since blood flow in the SMA $(124 \mathrm{ml} / \mathrm{m})$ did not increase significantly after its construction $(130 \mathrm{ml} / \mathrm{m})$. Histological
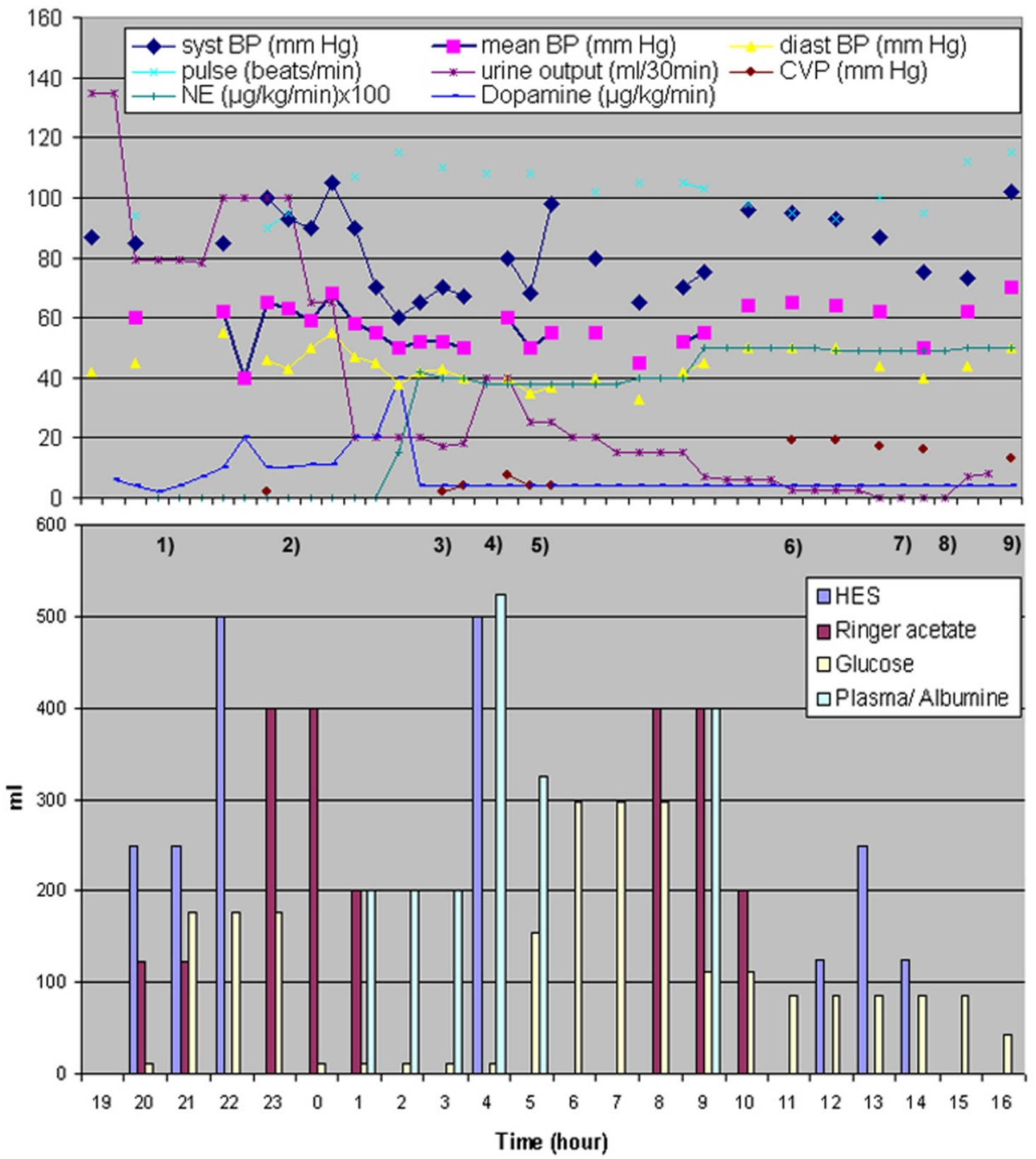

Fig. (4). Postoperative observations, pressor use, and volume substitution until laparotomy and intestinal resection. HES = hydroxyethyl starch, $\mathrm{NE}=$ norepinephrine, $\mathrm{CVP}=$ central venous pressure, 1) extubation, 2) feet cold, 3) urinary bladder pressure: $\left.10 \mathrm{~cm} \mathrm{H}_{2} \mathrm{O}, \mathbf{4}\right)$ feet tepid, 5) cardiac index: $5.4 \mathrm{~L} / \mathrm{min} / \mathrm{m}^{2}$; pulmonary artery wedged pressure: $7 \mathrm{~mm} \mathrm{Hg}, 6$ ) cardiac index: $4.7 \mathrm{~L} / \mathrm{min} / \mathrm{m}^{2}$; systemic vascular resistance: 390 dynes/sec x cm$~_{-5}$; mean pulmonary artery pressure: $25 \mathrm{~mm} \mathrm{Hg}, 7$ ) CT angiography was performed and he was reintubated, 8) feet cold, 9) start of laparotomy. 
examination did not show any atheroemboli in arteries of resected intestines. After intestinal resection Penicillin G was substituted for Ceftriaxone and Metronidazole.

Follow-up CT examinations showed that the thrombus in the origin of the SMA lysed spontaneously. Despite initial improvement and satisfactory findings, including a SMA blood flow of $440 \mathrm{ml} / \mathrm{m}$, at a second look laparotomy two days later, his further course was complicated with multiorgan failure and candida sepsis and he died after 32 days in the intensive care unit. Autopsy showed fungus in vessels from the lungs, liver and heart. The vascular prostheses and anastomoses were intact and without signs of infection.

\section{DISCUSSION}

In our case, by using a temporary bypass from the descending thoracic aorta to the aortic prosthesis, ischemic time, except in the celiac territory, was limited to the time needed to open the aneurysm, remove infected tissue and suture one anastomosis. The temporary bypass to the aortic prosthesis, furthermore, enabled the orthopedic surgeon to get ample access to the anterior aspect of the spine, while all vascular territories were perfused. After completion of the proximal anastomosis, the osteomyelitic focus in the spine became virtually inaccessible. Bypassing all four visceral vessels combined with an axillofemoral bypass or with a stentgraft and followed by removal of infected aneurysm tissue was considered, but was not thought to be a safer option.

Postoperative hypotension in combination with high doses of catecholamines is a likely cause of the intestinal infarction in our case [9]. Postoperative hypotension was likely caused by the initial clamp ischemia and uncontrolled infection in the extensive aneurysm sac and the surgical trauma were possible contributory causes [10]. Although 45 minutes of intestinal ischemia due to aortic clamping does not normally cause irreversible intestinal injury, it induces a systemic inflammatory response and a capillary leak syndrome [11] with a sizable fluid shift to interstitial space which may necessitate aggressive volume replacement to maintain blood pressure. Since the patient had biliary cirrhosis, some degree of portal hypertension most likely was present. Aortic clamping-induced visceral ischemia could have worsened this due to capillary leak-induced hepatic edema. Portal hypertension also may have reduced collateral intestinal perfusion, thus aggravating the clampinduced ischemia. With a mean $\mathrm{BP}$ of $50 \mathrm{~mm} \mathrm{Hg}$, an abdominal pressure of $10 \mathrm{~cm} \mathrm{H}_{2} \mathrm{O}$, a CVP of $1-3 \mathrm{~mm} \mathrm{Hg}$, and a portal venous pressure of $5-10 \mathrm{~mm} \mathrm{Hg}$, intestinal perfusion pressure could have been in the range of 30-37 mm $\mathrm{Hg}$, which may not be enough to maintain aerobic metabolism [12]. Aerobic metabolism may be essential in the period after clamp ischemia to allow restitution of the ischemic intestines. Presumably, as insufficient perfusion pressure gradually rendered the intestinal ischemia irreversible postoperatively, intestinal gangrene contributed ever more to the septic circulation, gradually changing from being mostly an effect to being both cause and effect of the hypotension.

Atheroembolism seems an unlikely cause of the intestinal ischemia, since there were no atheroemboli in arteries of the resected intestines. Since atheroembolism is a potential risk with the use of a side-biting clamp on the descending aorta, however, in future utilization of the method, it may be safer to divert blood to the shunt from an axillary artery. Air embolism could have been a cause if air was not completely evacuated from the graft construct prior to completion of the distal and celiac anastomoses. Finally, the ischemic injury may have been due to a combination of any of the aforementioned causes and the initial clamp ischemia.

The non-occluding thrombus in the SMA ostium may have formed in situ due to low flow e.g. during hypovolemia and pressor use, or it may have been an embolus originating from e.g. thrombus build-up in the creases of the ligated prosthesis construct. Adequate flushing to get rid of any thrombi after removal of temporary ligatures on vascular prostheses may be important.

\section{CONCLUSION}

Although not a method proven by success in the present case, during operations for ITAA, ischemic time during aortic clamping may be reduced by the time needed for the proximal anastomosis by using a presutured temporary shunt to the permanent aortic prosthesis. The shunt may be used to avoid ischemic injury in selected patients, especially in patients who need to be operated on despite severe infection or hemorrhagic diathesis, in which case it may be desirable to avoid the use of conventional extracorporal circulation. Adequate measures have to be taken to avoid embolism of air or thrombus from the vascular graft construct, and the axillary artery might be preferable for supplying blood to the shunt in patients with aortic atheromas to exclude the possibility of atheroembolism by the use of a side-biting clamp on the descending aorta.

\section{ACKNOWLEDGEMENTS}

ED planned and performed the vascular operations on the patient and wrote the manuscript. AA performed the orthopedic part of the operations and revised the manuscript.

Audun Braaten ${ }^{1}$, MD, processed the CT image used for Fig. (1).

Svein Amundsen ${ }^{2}$, MD, participated in the main operation and Aly Dicho, ${ }^{3}$ MD, performed intestinal resection and assisted during performance of the aorta-SMA bypass. Hans Flaatten ${ }^{4}, \mathrm{MD}, \mathrm{PhD}$, read the manuscript and agreed to the description of the postoperative treatment. Peer Lilleng 5 , MD, $\mathrm{PhD}$, scrutinized the histological sections, looking for atheroemboli in resected intestines.

${ }^{1}$ Department of Radiology, ${ }^{2}$ Department of Vascular Surgery, ${ }^{3}$ Department of Gastroenterological and Acute Surgery, ${ }^{4}$ Department of Anesthesiology, ${ }^{5}$ Department of Pathology, all at Haukeland University Hospital, Bergen, Norway.

\section{CONSENT}

Written informed consent was obtained from the next of kin of the patient for publication of this case report and accompanying images.

\section{ABBREVIATIONS}

NSAIDs $=$ Non-steroidal anti-inflammatory drugs 


$$
\begin{array}{lll}
\text { SMA } & =\text { Superior mesenteric artery } \\
\text { PTFE } & =\text { Polytetrafluoroethylene } \\
\text { CPAP } & =\begin{array}{l}
\text { Continuous positive airway pressure } \\
\text { ventilation }
\end{array} \\
\text { PEEP } & =\text { Peak end expiratory pressure } \\
\text { CT } & =\text { Computer tomography } \\
\text { BP } & =\text { Blood pressure }
\end{array}
$$

\section{REFERENCES}

[1] Chen IM, Chang HH, Hsu CP, et al. Ten-year experience with surgical repair of mycotic aortic aneurysms. J Chin Med Assoc 2005; 68(6): 265-71.

[2] Fillmore AJ, Valentine RJ. Surgical mortality in patients with infected aortic aneurysms. J Am Coll Surg 2003; 196(3): 435-41.

[3] Muller BT, Wegener OR, Grabitz K, et al. Mycotic aneurysms of the thoracic and abdominal aorta and iliac arteries: experience with anatomic and extra-anatomic repair in 33 cases. J Vasc Surg 2001; 33(1): 106-13.

[4] Reddy DJ, Shepard AD, Evans JR, et al. Management of infected aortoiliac aneurysms. Arch Surg 1991; 126(7): 873-8.

[5] Kieffer E, Chiche L, Godet G, et al. Type IV thoracoabdominal aneurysm repair: predictors of postoperative mortality, spinal cord injury, and acute intestinal ischemia. Ann Vasc Surg 2008; 22(6): 822-8.

[6] Conrad MF, Cambria RP. Contemporary management of descending thoracic and thoracoabdominal aortic aneurysms: endovascular versus open. Circulation 2008; 117(6): 841-52.

[7] Miller CC, III, Villa MA, Achouh P, et al. Intraoperative skeletal muscle ischemia contributes to risk of renal dysfunction following thoracoabdominal aortic repair. Eur J Cardiothorac Surg 2008; 33(4): 691-4.

[8] Edmunds LH. The evolution of cardiopulmonary bypass: lessons to be learned. Perfusion-Uk 2002; 17(4): 243-51.

[9] Krejci V, Hiltebrand LB, Sigurdsson GH. Effects of epinephrine, norepinephrine, and phenylephrine on microcirculatory blood flow in the gastrointestinal tract in sepsis. Crit Care Med 2006; 34(5): $1456-63$

[10] Zhao H, Spain DA, Matheson PJ, et al. Progressive decrease in constrictor reactivity of the non-absorbing intestine during chronic sepsis. Shock 2001; 16(1): 40-3.

[11] Poggetti RS, Moore FA, Moore EE, et al. Simultaneous liver and lung injury following gut ischemia is mediated by xanthine oxidase. J Trauma 1992; 32(6): 723-7

[12] Frojse R, Lehtipalo S, Winso O, et al. Assessment of graded intestinal hypoperfusion and reperfusion using continuous saline tonometry in a porcine model. Eur J Vasc Endovasc Surg 2004; 28(1): $79-88$

(c) Dregelid and Algaard; Licensee Bentham Open.

This is an open access article licensed under the terms of the Creative Commons Attribution Non-Commercial License (http://creativecommons.org/licenses/by$\mathrm{nc} / 3.0 /$ ) which permits unrestricted, non-commercial use, distribution and reproduction in any medium, provided the work is properly cited. 